\title{
NEITHER ASYMPTOTIC STABILITY NOR NONRECURRENCE IS A SUFFICIENT BASIS FOR THE SECOND LAW
}

\author{
BY \\ W. A. DAY \\ Hertford College, Oxford, England
}

1. Introduction. This paper is concerned with two assertions about the behaviour of isolated thermodynamic systems, viz.,

AsYMPTOTIC STABILITY. Isolated systems seek a dead level.

NONRECURRENCE. Un système isolé où se sont exercées des influences thermiques ne revient pas à un état antérieur.

The first of these is due to Bridgman [1, p. 116], while the second is due to Perrin [2, p. 63].

The question at issue here is whether asymptotic stability or nonrecurrence will serve as surrogate for any of the more familiar statements of the second law of thermodynamics that are associated with Clausius, Kelvin, or Planck. It would be a most interesting state of affairs if either assertion would so serve, for neither is framed directly in terms of the concepts of heat and work which occur in traditional statements of the second law.

Perrin certainly believed nonrecurrence to be equivalent to the second law for he said, "Je dois à M. Langevin la conviction où je suis que, sous l'une ou l'autre de ces formes, l'idée que je viens d'énoncer contient mieux que les énoncés ordinaires ce qu'il y a d'essentiel dans le second principe de la Thermodynamique, ou principe de Carnot..." [loc. cit.].

Bridgman did not claim so much for asymptotic stability, and he recognised that various formulations of the second law are not likely to be exactly equivalent. Nonetheless, he regarded asymptotic stability as the background against which the second law should be viewed, and, in the light of Perrin's claims for nonrecurrence, it is natural to ask if a hypothesis of asymptotic stability is strong enough to imply the second law. The expectation that an asymptotically stable system will have a Lyapunov function, from which its entropy might be constructed, suggests that the answer to this question may be an affirmative one.

My purpose is to show that, in fact, neither asymptotic stability nor nonrecurrence, whether taken separately or together, is sufficient to imply the second law. I shall do so by constructing constitutive relations which describe a thermoelastic fluid which exhibits both asymptotic stability and nonrecurrence but whose efficiency in a cyclic process exceeds what the second law permits. 
I have pointed out elsewhere [3,4] that nonrecurrence cannot imply the second law, but the arguments of both those references depend upon a linearization of the governing field equations which I shall not make here. It will transpire, though, that the temperature satisfies a linear partial differential equation even within our nonlinear theory.

2. Momentum, energy, and isolation. Our considerations throughout are entirely one-dimensional. To begin with, let us suppose the body to have a reference configuration in which it occupies an interval, $[A, B]$ say, of the real line $\mathbb{R}$. We shall label the particles of the body by the points they occupy when the body is placed in the reference configuration; thus the points $x, y, z, \ldots$ of $[A, B]$ are material, or Lagrangian, coordinates. In Sec. 4 the time $t$ takes its values in the set $\mathbb{R}^{+}$of nonnegative real numbers, but in Secs. 5 and 6, where we consider periodic processes, it takes its values in $\mathbb{R}$.

When the body is deformed the particle $x$ comes to occupy the point $x+u(x, t)$ of the real line at the time $t$, where $u$ is the displacement; of course, the point $x+u(x, t)$ need not belong to the interval $[A, B]$. The displacement is required to be twice continuously differentiable and to preserve the strict ordering of points, i.e., $x+u(x, t)<y+u(y, t)$ whenever $x$ and $y$ are points of $[A, B]$ with $x<y$. This requirement will be met by demanding a little more, viz., that the deformation gradient $1+E$ be positive, where $E=\partial u / \partial x$ is the strain.

It is proposed to set up balance laws for momentum and energy; the latter balance law is the form that the first law of thermodynamics takes in the present context.

Let us introduce the following fields: the mass density in the reference configuration $\rho(x)$, the pressure $p(x, t)$, the external force density $f(x, t)$, the internal energy density $U(x, t)$, the heat flux $q(x, t)$, and the external rate-of-heating density $h(x, t)$. Of these $\rho, f, h$ are required to be continuous, while $p, U, q$ are required to be continuously differentiable. Each of the densities $\rho, U, f, h$ is measured with respect to length in the reference configuration. The pressure is required to be strictly positive.

We postulate that, for each subinterval $[a, b]$ of $[A, B]$, the momentum balance law

$$
-[p]_{a}^{b}+\int_{a}^{b} f d x=\frac{d}{d t} \int_{a}^{b} \rho \frac{\partial u}{\partial t} d x
$$

is satisfied, and so is the energy balance law

$$
-\left[p \frac{\partial u}{\partial t}\right]_{a}^{b}+\int_{a}^{b} f \frac{\partial u}{\partial t} d x-[q]_{a}^{b}+\int_{a}^{b} h d x=\frac{d}{d t} \int_{a}^{b}\left(\frac{1}{2} \rho\left(\frac{\partial u}{\partial t}\right)^{2}+U\right) d x .
$$

Here, the symbol [ $]_{a}^{h}$ denotes the change in a field over the interval $[a, b]$; thus $[p]_{a}^{b}=\left.p\right|_{b}-\left.p\right|_{a}$, and so forth.

Straightforward, and standard, arguments enable us to conclude that the balance laws imply the momentum equation

$$
-\frac{\partial p}{\partial x}+f=\rho \frac{\partial^{2} u}{\partial t^{2}}
$$


the energy equation

$$
-\frac{\partial}{\partial x}\left(p \frac{\partial u}{\partial t}\right)+f \frac{\partial u}{\partial t}-\frac{\partial q}{\partial x}+h=\rho \frac{\partial u}{\partial t} \frac{\partial^{2} u}{\partial t^{2}}+\frac{\partial U}{\partial t},
$$

and the reduced energy equation

$$
-p \frac{\partial E}{\partial t}-\frac{\partial q}{\partial x}+h=\frac{\partial U}{\partial t}
$$

Conversely, if $u$ is twice continuously differentiable, if $p, U, q$ are continuously differentiable, if $\rho, f, h$ are continuous, and if $E=\partial u / \partial x$, the momentum equation and the energy equation, or, equivalently, the momentum equation and the reduced energy equation, ensure that the momentum balance law and the energy balance law are valid for each subinterval $[a, b]$ of $[A, B]$.

In view of these conclusions it will be enough for us to work with the momentum equation (2.1) and the reduced energy equation (2.2) rather than with the balance laws themselves.

The body is said to be mechanically isolated if the external force density $f \equiv 0$ and if the boundary conditions

$$
\left.\frac{\partial u}{\partial t}\right|_{A}=\left.\frac{\partial u}{\partial t}\right|_{B}=0
$$

are satisfied.

The body is said to be thermally isolated if the external rate-of-heating density $h \equiv 0$ and if the boundary conditions

$$
\left.q\right|_{A}=\left.q\right|_{B}=0
$$

are satisfied.

If the body is both mechanically isolated and thermally isolated we describe it as being isolated.

It is clear from the energy balance law, with $[a, b]$ chosen to be $[A, B]$, that the total energy

$$
\int_{A}^{B}\left(\frac{1}{2} \rho\left(\frac{\partial u}{\partial t}\right)^{2}+U\right) d x
$$

is constant if the body is isolated.

3. Constitutive relations. In order to specify a thermoelastic fluid we introduce the absolute temperature $\theta(x, t)$, whose values are strictly positive, and impose the constitutive relations

$$
\begin{gathered}
1+E=\varphi(p) \theta, \\
U=\psi(p) \varphi(p) \theta, \\
q=-k \frac{\partial \theta}{\partial x} .
\end{gathered}
$$

The last of these is Fourier's law; in our model it is the material temperature gradient which enters into the statement of that law. 
We place the following restrictions upon the response of the fluid:

(i) The mass density $\rho \equiv 0$.

(ii) The thermal conductivity $k$ is a strictly positive constant.

(iii) The functions $\varphi(p)$ and $\psi(p)$ are defined for every $p>0$, and each is strictly positive. Moreover, $\varphi$ is strictly decreasing, $\psi$ is strictly increasing, $\varphi(p) \rightarrow \infty$ and $\psi(p) \rightarrow 0$ when $p \rightarrow 0$, and $\varphi(p) \rightarrow 0$ and $\psi(p) \rightarrow \infty$ when $p \rightarrow \infty$.

The implication of the drastic hypothesis (i) is that the effects of inertia and of kinetic energy are ignored; I introduce this simplification for the sake of mathematical convenience, but clearly it would be highly desirable if it could be established that the main thrust of the conclusions remains valid even when inertia is taken into account.

Hypothesis (ii) ensures that heat conduction plays an important part in our considerations.

Hypothesis (iii), and the fact that $\theta>0$, ensure that $1+E>0$, as kinematic considerations require, and that $U>0$.

Henceforth, we require (i), (ii), (iii) to hold, but, as we shall see, such a requirement does not ensure that the thermoelastic fluid is compatible with the second law of thermodynamics.

4. Asymptotic stability and nonrecurrence. If, as (i) demands, $\rho \equiv 0$, and if $f \equiv 0$, the momentum equation $(2.1)$ reduces to the equation

$$
\frac{\partial p}{\partial x}=0
$$

Hence, the pressure is independent of $x$ in these circumstances, and may be written simply as $p(t)$. On substituting from the constitutive relations (3.1), (3.2), (3.3) into the reduced energy equation (2.2), setting $h \equiv 0$, and writing $\varphi^{\prime}=d \varphi / d p, \psi^{\prime}=$ $d \psi / d p$, and $\dot{p}=d p / d t$, we conclude that, when $f \equiv 0$ and $h \equiv 0$, the pressure $p(t)$ is a function of $t$ only, and the temperature $\theta(x, t)$ satisfies the parabolic differential equation

$$
k \frac{\partial^{2} \theta}{\partial x^{2}}=(\psi(p)+p) \varphi(p) \frac{\partial \theta}{\partial t}+\left(\psi^{\prime}(p) \varphi(p)+(\psi(p)+p) \varphi^{\prime}(p)\right) \dot{p} \theta
$$

In view of hypothesis (iii), the (possibly variable) coefficient $(\psi(p)+p) \phi(p)$ is always positive.

Up to this point we have made no use of the boundary conditions. Our next result assumes the thermal boundary conditions $(2.4)$ to be satisfied, but the mechanical conditions (2.3) need not hold. Because of Fourier's law (3.3), the conditions (2.4) are equivalent to the conditions

$$
\left.\frac{\partial \theta}{\partial x}\right|_{A}=\left.\frac{\partial \theta}{\partial x}\right|_{B}=0
$$

We shall put

$$
\Delta=\log ((\psi(p)+p) \varphi(p) \theta)-\int^{p} \frac{d \lambda}{\psi(\lambda)+\lambda},
$$


and prove that, if $f \equiv 0$, and if the body is thermally isolated, the integral

$$
\int_{A}^{B} \Delta d x
$$

is an increasing function of $t$.

This result, together with our proof of asymptotic stability which will be given presently, provides what Bridgman [1, p. 120] refers to as a "numerical measure of the degree of progiess which a system has made in the direction towards its dead level," and it does so even when the body is not compatible with the second law.

To verify the assertion, we calculate the derivative of $\Delta$ with respect to $t$ and find it to be

$$
\begin{aligned}
\frac{\partial \Delta}{\partial t} & =\frac{1}{\theta} \frac{\partial \theta}{\partial t}+\frac{\left[\left(\psi^{\prime}(p)+1\right) \varphi(p)+(\psi(p)+p) \varphi^{\prime}(p)\right]}{(\psi(p)+p) \varphi(p)} \dot{p}-\frac{\dot{p}}{\psi(p)+p} \\
& =\frac{1}{\theta} \frac{\partial \theta}{\partial t}+\frac{\left[\psi^{\prime}(p) \varphi(p)+(\psi(p)+p) \varphi^{\prime}(p)\right]}{(\psi(p)+p) \varphi(p)} \dot{p} .
\end{aligned}
$$

It now follows from (4.1) that

$$
\begin{aligned}
\frac{\partial \Delta}{\partial t} & =\frac{k}{(\psi(p)+p) \varphi(p)} \cdot \frac{1}{\theta} \frac{\partial^{2} \theta}{\partial x^{2}} \\
& =\frac{k}{(\psi(p)+p) \varphi(p)}\left[\frac{\partial}{\partial x}\left(\frac{1}{\theta} \frac{\partial \theta}{\partial x}\right)+\frac{1}{\theta^{2}}\left(\frac{\partial \theta}{\partial x}\right)^{2}\right] .
\end{aligned}
$$

Thus, on integrating with respect to $x$, using the fact that $p$ is independent of $x$, and appealing to the boundary conditions (4.2), we deduce that

$$
\frac{d}{d t} \int_{A}^{B} \Delta d x=\int_{A}^{B} \frac{\partial \Delta}{\partial t} d x=\frac{k}{(\psi(p)+p) \varphi(p)} \int_{A}^{B} \frac{1}{\theta^{2}}\left(\frac{\partial \theta}{\partial x}\right)^{2} d x \geq 0,
$$

which proves the result.

We now establish asymptotic stability by showing that, when the body is isolated, the pressure $p$ is independent of both $x$ and $t$, and, as $t \rightarrow \infty$, the temperature and the strain converge to limits that are independent of $x$.

In order to verify this, let us introduce the initial temperature $\theta_{0}(x)=\theta(x, 0)(x \in$ $[A, B])$ and its mean value

$$
\mu=\frac{1}{B-A} \int_{A}^{B} \theta_{0}(x) d x .
$$

If the body is isolated, the thermal boundary conditions (2.4), or equivalently the conditions (4.2), must hold, and so must the mechanical conditions (2.3). According to this last, the boundary points $A$ and $B$ are displaced to points, $a$ and $b$ say, which do not vary with $t$. Hence (2.3) can be restated in the form

$$
\left.u\right|_{A}=a-A,\left.\quad u\right|_{B}=b-B .
$$


On integrating the constitutive relation (3.1), and remembering that

$$
\begin{aligned}
\int_{A}^{B}(1+E) d x & =\int_{A}^{B}\left(1+\frac{\partial u}{\partial x}\right) d x \\
& =B-A+[u]_{A}^{B} \\
& =b-a,
\end{aligned}
$$

we see that

$$
b-a=\varphi(p(t)) \int_{A}^{B} \theta(x, t) d x,
$$

while, on integrating the constitutive relation (3.2), we see that

$$
\int_{A}^{B} U d x=\psi(p(t)) \varphi(p(t)) \int_{A}^{B} \theta(x, t) d x
$$

Hence,

$$
\psi(p(t))=\frac{1}{b-a} \int_{A}^{B} U d x .
$$

However, we know (since the body is isolated and $\rho \equiv 0$ ) that the total internal energy

$$
\int_{A}^{B} U d x
$$

is constant, and that constant must be strictly positive because $U$ is strictly positive. Since hypothesis (iii) ensures that $\psi$ is a bijection of the set of strictly positive numbers, (4.4) shows that the pressure $p$ must be independent of $t$. According to (4.3), therefore, the integral

$$
\int_{A}^{B} \theta d x
$$

must be independent of $t$ and, hence, must equal its initial value

$$
\int_{A}^{B} \theta_{0} d x=(B-A) \mu \text {. }
$$

It follows from (4.3) that

$$
b-a=\varphi(p)(B-A) \mu
$$

and, hence, that the pressure

$$
p=\varphi^{-1}\left(\frac{b-a}{(B-A) \mu}\right) .
$$

Because $p$ is constant, the differential equation (4.1) reduces to a heat equation

$$
k \frac{\partial^{2} \theta}{\partial x^{2}}=(\psi(p)+p) \varphi(p) \frac{\partial \theta}{\partial t},
$$

with constant coefficients, and a standard argument enables us to conclude from this and the boundary conditions (4.2) that

$$
\theta(x, t) \rightarrow \mu \quad \text { as } t \rightarrow \infty .
$$


It now follows from $(3.1)$ that

$$
1+E(x, t) \rightarrow \varphi(p) \mu=\frac{b-a}{B-A} \quad \text { as } t \rightarrow \infty,
$$

and, hence, the proof of asymptotic stability is complete.

It should be remarked that the rate of decay of the temperature is

$$
\theta(x, t)=\mu+O\left(\exp \left(\frac{-k \pi^{2} t}{(\psi(p)+p) \varphi(p)(B-A)^{2}}\right)\right)
$$

where

$$
(\psi(p)+p) \varphi(p)=\left[\psi\left(\varphi^{-1}\left(\frac{b-a}{(B-A) \mu}\right)\right)+\varphi^{-1}\left(\frac{b-a}{(B-A) \mu}\right)\right]\left(\frac{b-a}{(B-A) \mu}\right) .
$$

Thus, although the temperature satisfies a linear partial differential equation, the rate at which the temperature decays to its limiting value depends upon the mean initial temperature $\mu$ and the ratio $(b-a) /(B-A)$ of the deformed length of the body to its reference length.

It is also clear from standard results about the heat equation, subject to the boundary conditions (4.2), that the integral

$$
\int_{A}^{B}(\theta-\mu)^{2} d x
$$

is a strictly decreasing function of $t$, unless the initial temperature $\theta_{0}(x)$ is independent of $x$, in which case $\theta(x, t) \equiv \mu$. In this way we can immediately deduce a proposition about nonrecurrence: if the body is isolated, and if the initial temperature is not identically constant, it is impossible that there exist distinct times $t_{1}$ and $t_{2}$ such that $\theta\left(x, t_{1}\right) \equiv \theta\left(x, t_{2}\right)$ for all $x \in[A, B]$.

5. Incompatibility with the second law. In order that the fluid whose constitutive relations are $(3.1),(3.2),(3.3)$ be compatible with the second law, it is necessary that there exist a Gibbs free energy density $G(p, \theta)$ and an entropy density $S(p, \theta)$ such that

$$
G=U+p E-\theta S, \quad E=\left(\frac{\partial G}{\partial p}\right)_{\theta}, \quad S=-\left(\frac{\partial G}{\partial \theta}\right)_{p} .
$$

Thus

$$
U=G-p\left(\frac{\partial G}{\partial p}\right)_{\theta}-\theta\left(\frac{\partial G}{\partial \theta}\right)_{p},
$$

and, on differentiating with respect to $p$, we see that the condition

$$
\left(\frac{\partial U}{\partial p}\right)_{\theta}+p\left(\frac{\partial E}{\partial p}\right)_{\theta}+\theta\left(\frac{\partial E}{\partial \theta}\right)_{p}=0
$$

is necessary for compatibility with the second law. If we substitute from (3.1) and (3.2) into (5.2), and cancel a factor $\theta$ from each side, we find that (5.2) is equivalent to the equation

$$
(\psi(p) \varphi(p))^{\prime}+p \varphi^{\prime}(p)+\varphi(p)=0
$$


Hence the product $(\psi(p)+p) \varphi(p)$, which is the coefficient of $\partial \theta / \partial t$ in the parabolic equation (4.1), must be constant (i.e., independent of the presssure $p$ ). If we call this constant $c$, integration of the equations (5.1) tells us that

$$
\begin{aligned}
& G=\theta \int^{p} \varphi(\lambda) d \lambda-c(\theta \log \theta-\theta), \\
& S=-\int^{p} \varphi(\lambda) d \lambda+c \log \theta,
\end{aligned}
$$

and, therefore, that the specific heat at constant pressure, which is $\theta(\partial S / \partial \theta)_{p}$, coincides with $c$. In summary: a fluid whose constitutive relations are (3.1), (3.2), (3.3) cannot be compatible with the second law unless

$$
c=(\psi(p)+p) \varphi(p)
$$

is independent of $p$, in which case $c$ is the specific heat at constant pressure.

Our hypothesis (iii), of course, does not force $c$ to be independent of $p$, and therefore we have shown that it is possible for the fluid to exhibit both asymptotic stability and nonrecurrence and yet to be incompatible with the second law, in the sense that the condition (5.2) is violated.

6. More on incompatibility with the second law. We turn finally to showing that it is possible for the fluid to exhibit both asymptotic stability and nonrecurrence and to violate a condition which has a more immediate physical interpretation than does (5.2), and which is framed in terms of the concepts of heat and work which enter into traditional statements of the second law.

To this end, we suppose no longer that the body is isolated but rather is immersed in a heat bath at constant temperature $\tau$, while the boundary points $A$ and $B$ are subjected to a variable pressure $p(t)$ which is periodic in $t$, with period $T$, say. Thus, it is supposed that $f \equiv 0$ and $h \equiv 0$ and, hence, that the temperature $\theta(x, t)$ satisfies the parabolic equation (4.1). It is supposed, furthermore, that $\theta$ satisfies the boundary conditions

$$
\left.\theta\right|_{A}=\left.\theta\right|_{B}=\tau
$$

and that $\theta$ and $p$ are periodic in $t$, with period $T$, i.e., $\theta(x, t+T)=\theta(x, t)$ and $p(t+T)=p(t)$ for every $x \in[A, B]$ and every $t \in \mathbb{R}$.

The net heat flux into the body is

$$
Q=-[q]_{A}^{B},
$$

and the rate at which the body does work on its exterior is

$$
W=\left[p \frac{\partial u}{\partial t}\right]_{A}^{B}
$$

On returning to the energy balance law, and setting $[a, b]=[A, B], f \equiv 0, h \equiv 0$, and $\rho \equiv 0$, we see that

$$
-W+Q=\frac{d}{d t} \int_{A}^{B} U d x
$$


Thus, on integrating with respect to $t$, and appealing to the periodicity of $p$ and $\theta$ (and hence of $U$ ) we deduce, as we should expect, that

$$
\int_{0}^{T} W d t=\int_{0}^{T} Q d t
$$

i.e., the work done by the body in a period coincides with the net heat absorbed by it in a period.

Next, let us observe that the differential equation (4.1) implies that

$$
\begin{aligned}
-\frac{\partial}{\partial x}\left(\frac{1}{\theta} q\right)= & k \frac{\partial}{\partial x}\left(\frac{1}{\theta} \frac{\partial \theta}{\partial x}\right) \\
= & \frac{k}{\theta} \frac{\partial^{2} \theta}{\partial x^{2}}-\frac{k}{\theta^{2}}\left(\frac{\partial \theta}{\partial x}\right)^{2} \\
= & (\psi(p)+p) \varphi(p) \frac{1}{\theta} \frac{\partial \theta}{\partial t} \\
& +\left(\psi^{\prime}(p) \varphi(p)+(\psi(p)+p) \varphi^{\prime}(p)\right) \dot{p}-\frac{k}{\theta^{2}}\left(\frac{\partial \theta}{\partial x}\right)^{2} .
\end{aligned}
$$

On integrating with respect to $x$, and appealing to the boundary conditions (6.1), we see that

$$
\begin{aligned}
\frac{Q}{\tau}= & (\psi(p)+p) \varphi(p) \frac{d}{d t} \int_{A}^{B} \log \theta d x \\
& +(B-A)\left(\psi^{\prime}(p) \varphi(p)+(\psi(p)+p) \varphi^{\prime}(p)\right) \dot{p}-k \int_{A}^{B} \frac{1}{\theta^{2}}\left(\frac{\partial \theta}{\partial x}\right)^{2} d x .
\end{aligned}
$$

If now we suppose the body to be compatible with thermodynamics, and, hence, $c=(\psi(p)+p) \varphi(p)$ to be independent of $p$, and if we integrate with respect to $t$ and appeal to periodicity we deduce that

$$
\frac{1}{\tau} \int_{0}^{T} Q d t=-k \int_{0}^{T} \int_{A}^{B} \frac{1}{\theta^{2}}\left(\frac{\partial \theta}{\partial x}\right)^{2} d x d t \leq 0 .
$$

Since $\tau$ is positive, it follows, with the aid of (6.2), that

$$
\int_{0}^{T} W d t \leq 0
$$

Thus, granted that the body is immersed in a heat bath at constant temperature and that $f \equiv 0$ and $h \equiv 0$, we have shown that if the body is compatible with thermodynamics it can never perform a positive amount of work in a periodic process, no matter how the pressure is adjusted.

What we shall show now is that it is possible for the body to meet our hypotheses (i), (ii), (iii), and, hence, to exhibit asymptotic stability and nonrecurrence, but yet to violate this last conclusion in that the body can perform a positive amount of work on its exterior when it is immersed in a heat bath at constant temperature and undergoes a periodic process. To accomplish this we shall establish that if there is a $p_{0}>0$ for which

$$
\psi^{\prime}\left(p_{0}\right) \varphi\left(p_{0}\right)+\left(\psi\left(p_{0}\right)+p_{0}\right) \varphi^{\prime}\left(p_{0}\right)>0
$$


it is possible to choose the periodically varying pressure so that the work done by the body in a period is strictly positive.

Two remarks should be made about the condition (6.3). Firstly, there are $\varphi(p)$ and $\psi(p)$ which satisfy both the hypothesis (iii) and the inequality (6.3). An example is provided by taking

$$
\varphi(p)=\frac{\varphi_{0}}{p}, \quad \psi(p)=\psi_{0} p^{2},
$$

where $\varphi_{0}$ and $\psi_{0}$ are any strictly positive constants; in this case (6.3) holds for every $p_{0}>1 / \psi_{0}$. Secondly, (6.3) cannot hold if the body is compatible with thermodynamics for then the product $(\psi(p)+p) \varphi(p)$ is independent of $p$. On setting the derivative of the product with respect to $p$ equal to zero, we have

$$
\psi^{\prime}(p) \varphi(p)+(\psi(p)+p) \varphi^{\prime}(p)=-\varphi(p)<0,
$$

and so (6.3) cannot hold.

In order to begin the proof of our claim let us recall that the temperature satisfies the parabolic equation (4.1), which we rewrite as

$$
k \frac{\partial^{2} \theta}{\partial x^{2}}=r(t) \frac{\partial \theta}{\partial t}+\dot{s}(t) \theta
$$

where

$$
\begin{aligned}
& r(t)=(\psi(p(t))+p(t)) \varphi(p(t)) \\
& s(t)=\psi(p(t)) \varphi(p(t))+\int^{p(t)} \lambda \varphi^{\prime}(\lambda) d \lambda .
\end{aligned}
$$

We recall too that the temperature satisfies the boundary conditions (6.1).

The main technical step in the argument is to check that if

$$
\operatorname{Max}\left|\frac{1}{2} \dot{r}-\dot{s}\right|<\left(\frac{\pi}{B-A}\right)^{2} k,
$$

the work done by the body in a period cannot be less than

$$
\begin{aligned}
\frac{\tau}{12 k}(B-A)^{3} \int_{0}^{T}\left(\dot{r} \dot{s}-\dot{s}^{2}\right) d t & \\
& -\frac{(\tau / 2 \sqrt{30} k)(B-A)^{3}\left(\int_{0}^{T}(\dot{r}-\dot{s})^{2} d t \int_{0}^{T}\left(r \ddot{s}+\dot{s}^{2}\right)^{2} d t\right)^{1 / 2}}{(\pi /(B-A))^{2} k-\operatorname{Max}\left|\frac{1}{2} \dot{r}-\dot{s}\right|} .
\end{aligned}
$$

Let us put

$$
\begin{aligned}
& \xi(x, t)=\theta(x, t)-\tau+\frac{\tau}{2 k}(x-A)(B-x) \dot{s}(t), \\
& g(x, t)=\frac{\tau}{2 k}(x-A)(B-x)\left(r(t) \ddot{s}(t)+\dot{s}(t)^{2}\right) .
\end{aligned}
$$

Then it follows from Eq. (6.4) that $\xi$ satisfies the inhomogeneous equation

$$
k \frac{\partial^{2} \xi}{\partial x^{2}}=r \frac{\partial \xi}{\partial t}+\dot{s} \xi-g .
$$


while, by virtue of the boundary conditions $(6.1), \xi$ satisfies the homogeneous boundary conditions

$$
\left.\xi\right|_{A}=\left.\xi\right|_{B}=0 .
$$

The net heat flux into the body is

$$
\begin{aligned}
Q= & k\left[\frac{\partial \theta}{\partial x}\right]_{A}^{B} \\
= & k \int_{A}^{B} \frac{\partial^{2} \theta}{\partial x^{2}} d x \\
= & \int_{A}^{B}\left(\tau \dot{s}+k \frac{\partial^{2} \xi}{\partial x^{2}}\right) d x \\
= & (B-A) \tau \dot{s}+\int_{A}^{B}\left(r \frac{\partial \xi}{\partial t}+\dot{s} \xi-g\right) d x \\
= & (B-A) \tau \dot{s}+\int_{A}^{B}\left(r \frac{\partial \xi}{\partial t}+\dot{s} \xi\right) d x-\frac{\tau}{12 k}(B-A)^{3}\left(r \ddot{s}+\dot{s}^{2}\right) \\
= & \frac{d}{d t}\left[(B-A) \tau s+\int_{A}^{B} r \xi d x-\frac{\tau}{12 k}(B-A)^{3} r \dot{s}\right] \\
& +\int_{A}^{B}(\dot{s}-\dot{r}) \xi d x+\frac{\tau}{12 k}(B-A)^{3}\left(\dot{r} \dot{s}-\dot{s}^{2}\right) .
\end{aligned}
$$

Thus, if we integrate with respect to $t$ and appeal to periodicity and to the equality of the work done and the net heat absorbed (6.2), we find the work done in a period to be

and, therefore,

$$
\int_{0}^{T} W d t=\frac{\tau}{12 k}(B-A)^{3} \int_{0}^{T}\left(\dot{r} \dot{s}-\dot{s}^{2}\right) d t+\int_{0}^{T} \int_{A}^{B}(\dot{s}-\dot{r}) \xi d x d t,
$$

$$
\begin{aligned}
\int_{0}^{T} W d t \geq & \frac{\tau}{12 k}(B-A)^{3} \int_{0}^{T}\left(\dot{r} \dot{s}-\dot{s}^{2}\right) d t \\
& -\left(\int_{0}^{T} \int_{A}^{B}(\dot{r}-\dot{s})^{2} d x d t \int_{0}^{T} \int_{A}^{B} \xi^{2} d x d t\right)^{(1 / 2)},
\end{aligned}
$$

by an appeal to the Schwarz inequality.

The next step is to estimate the integral involving $\xi$ which occurs in (6.9). According to the differential equation (6.7),

$$
\begin{aligned}
k \frac{\partial}{\partial x}\left(\xi \frac{\partial \xi}{\partial x}\right) & =k\left(\frac{\partial \xi}{\partial x}\right)^{2}+r \xi \frac{\partial \xi}{\partial t}+\dot{s} \xi^{2}-g \xi \\
& =k\left(\frac{\partial \xi}{\partial x}\right)^{2}+\frac{1}{2} \frac{\partial}{\partial t}\left(r \xi^{2}\right)+\left(\dot{s}-\frac{1}{2} \dot{r}\right) \xi^{2}-g \xi
\end{aligned}
$$

Thus, if we integrate with respect to $x$, and invoke the boundary conditions (6.8), we find that

$$
0=k \int_{A}^{B}\left(\frac{\partial \xi}{\partial x}\right)^{2} d x+\frac{1}{2} \frac{d}{d t} \int_{A}^{B} r \xi^{2} d x+\left(\dot{s}-\frac{1}{2} \dot{r}\right) \int_{A}^{B} \xi^{2} d x-\int_{A}^{B} g \xi d x .
$$


However, the boundary conditions also imply the inequality

$$
\int_{A}^{B}\left(\frac{\partial \xi}{\partial x}\right)^{2} d x \geq\left(\frac{\pi}{B-A}\right)^{2} \int_{A}^{B} \xi^{2} d x
$$

and so we have

$$
0 \geq \int_{A}^{B}\left[\left(\frac{\pi}{B-A}\right)^{2} k+\dot{s}-\frac{1}{2} \dot{r}\right] \xi^{2} d x+\frac{1}{2} \frac{d}{d t} \int_{A}^{B} r \xi^{2} d x-\int_{A}^{B} g \xi d x .
$$

A further integration with respect to $t$, and an appeal to periodicity and to the hypothesis $(6.5)$, now show that

$$
\begin{aligned}
\int_{0}^{T} \int_{A}^{B} g \xi d x d t & \geq \int_{0}^{T} \int_{A}^{B}\left[\left(\frac{\pi}{B-A}\right)^{2} k+\dot{s}-\frac{1}{2} \dot{r}\right] \xi^{2} d x d t \\
& \geq\left[\left(\frac{\pi}{B-A}\right)^{2} k-\operatorname{Max}\left|\frac{1}{2} \dot{r}-\dot{s}\right|\right] \int_{0}^{T} \int_{A}^{B} \xi^{2} d x d t .
\end{aligned}
$$

Thus, if we estimate the integral involving $g$ with the aid of the Schwarz inequality we deduce that

$$
\begin{aligned}
\int_{0}^{T} \int_{A}^{B} \xi^{2} d x d t & \leq \frac{\int_{0}^{T} \int_{A}^{B} g^{2} d x d t}{\left[(\pi /(B-A))^{2} k-\operatorname{Max}\left|\frac{1}{2} \dot{r}-\dot{s}\right|\right]^{2}} \\
& =\frac{(\tau / 2 k)^{2} \cdot\left((B-A)^{5} / 30\right) \int_{0}^{T}\left(r \ddot{s}+\dot{s}^{2}\right)^{2} d t}{\left[(\pi /(B-A))^{2} k-\operatorname{Max}\left|\frac{1}{2} \dot{r}-\dot{s}\right|\right]^{2}}
\end{aligned}
$$

and when we combine this last inequality with (6.9) we deduce, as claimed, that the work done cannot be less than the expression (6.6).

From this point on our method will be to consider a suitable family of "retarded" pressures and show that it is possible to arrange that the first term in the difference (6.6) is positive, while the second term is negligible by comparison with the first.

Because $\varphi$ and $\psi$ are continuously differentiable, (6.3) permits us to choose a $\delta>0$ such that

$$
\psi^{\prime}(p) \varphi(p)+(\psi(p)+p) \varphi^{\prime}(p)>0
$$

for every $p \in\left(p_{0}-\delta, p_{0}+\delta\right)$. Next, we choose the pressure $p(t)$ in such a way that $p(t)$ is nonconstant, $p(t)$ is periodic with some period $T$, and $p(t) \in\left(p_{0}-\delta, p_{0}+\delta\right)$ for every $t \in \mathbb{R}$. Now let $\varepsilon>0$, let us introduce the retardations

$$
\begin{aligned}
& p_{\varepsilon}(t)=p(\varepsilon t), \\
& r_{\varepsilon}(t)=r(\varepsilon t)=(\psi(p(\varepsilon t))+p(\varepsilon t)) \varphi(p(\varepsilon t)), \\
& s_{\varepsilon}(t)=s(\varepsilon t)=\psi(p(\varepsilon t)) \varphi(p(\varepsilon t))+\int^{p(\varepsilon t)} \lambda \varphi^{\prime}(\lambda) d \lambda,
\end{aligned}
$$

and let us consider the work done by the body in the retarded period $T / \varepsilon$ and ask what happens in the limits $\varepsilon \rightarrow 0$.

We have

$$
\left|\frac{1}{2} \dot{r}_{\varepsilon}(t)-\dot{s}_{\varepsilon}(t)\right|=\varepsilon\left|\frac{1}{2} \dot{r}(\varepsilon t)-\dot{s}(\varepsilon t)\right| \leq \varepsilon \operatorname{Max}\left|\frac{1}{2} \dot{r}-\dot{s}\right|,
$$


and, hence, it is certainly the case that

$$
\operatorname{Max}\left|\frac{1}{2} \dot{r}_{\varepsilon}-\dot{s}_{\varepsilon}\right|<\left(\frac{\pi}{B-A}\right)^{2} k
$$

if $\varepsilon$ is sufficiently small. Moreover,

$$
\begin{aligned}
\int_{0}^{T / \varepsilon}\left(\dot{r}_{\varepsilon} \dot{s}_{\varepsilon}-\dot{s}_{\varepsilon}^{2}\right) d t & =\int_{0}^{T / \varepsilon} \varepsilon^{2}\left(\dot{r}(\varepsilon t) \dot{s}(\varepsilon t)-\dot{s}(\varepsilon t)^{2}\right) d t \\
& =\varepsilon \int_{0}^{T}\left(\dot{r} \dot{s}-\dot{s}^{2}\right) d t,
\end{aligned}
$$

and, similarly,

$$
\begin{gathered}
\int_{0}^{T / \varepsilon}\left(\dot{r}_{\varepsilon}-\dot{s}_{\varepsilon}\right)^{2} d t=\varepsilon \int_{0}^{T}(\dot{r}-\dot{s})^{2} d t, \\
\int_{0}^{T / \varepsilon}\left(r_{\varepsilon} \ddot{s}_{\varepsilon}+\dot{s}_{\varepsilon}^{2}\right)^{2} d t=\varepsilon^{3} \int_{0}^{T}\left(r \ddot{s}+\dot{s}^{2}\right)^{2} d t .
\end{gathered}
$$

It now follows from (6.6) that the work done by the body in the retarded period $T / \varepsilon$ cannot be less than

$$
\frac{\varepsilon \tau}{12 k}(B-A)^{3} \int_{0}^{T}\left(\dot{r} \dot{s}-\dot{s}^{2}\right) d t-O\left(\varepsilon^{2}\right)
$$

and is, therefore, strictly positive provided that $\varepsilon$ is sufficiently small and

$$
\int_{0}^{T}\left(\dot{r} \dot{s}-\dot{s}^{2}\right) d t>0
$$

However,

$$
\begin{aligned}
& \dot{r}=\left[\left(\psi^{\prime}(p)+1\right) \varphi(p)+(\psi(p)+p) \varphi^{\prime}(p)\right] \dot{p}, \\
& \dot{s}=\left[\psi^{\prime}(p) \varphi(p)+(\psi(p)+p) \varphi^{\prime}(p)\right] \dot{p}, \\
& \dot{r}-\dot{s}=\varphi(p) \dot{p}
\end{aligned}
$$

and, therefore,

$$
\begin{aligned}
\dot{r} \dot{s}-\dot{s}^{2} & =(\dot{r}-\dot{s}) \dot{s} \\
& =\varphi(p)\left(\psi^{\prime}(p) \varphi(p)+(\psi(p)+p) \varphi^{\prime}(p)\right) \dot{p}^{2} .
\end{aligned}
$$

In view of the inequality (6.10), and the way in which $p(t)$ has been chosen, it follows that $(6.11)$ does hold and so the proof is complete.

\section{REFERENCES}

[1] P. W. Bridgman. The Nature of Thermodynamics, Harper Torchbooks, New York, 1961

[2] J. Perrin, Oeuvres Scientifiques de Jean Perrin, Editions CNRS, Paris, 1950

[3] W. A. Day, A comment on a formulation of the second law of thermodynamics, Arch. Rational Mech. Anal. 98, 211-227 (1987)

[4] W. A. Day, A Commentary on Thermodynamics, Springer Tracts in Natural Philosophy, Vol. 32. Springer-Verlag, New York, 1988 